\title{
Lactobacillus jensenii
}

National Cancer Institute

\section{Source}

National Cancer Institute. Lactobacillus jensenii. NCI Thesaurus. Code C86480.

A species of facultatively anaerobic, Gram positive, rod shaped bacteria in the phylum Firmicutes. This species is oblig ately homofermentative, requires pyridoxal for growth in culture and ferments trehalose and esculin but not lactose. L. jensenii is a colonizer of the healthy vag inal tract and inhibits the growth of gonococcal org anisms therein, but may be pathogenic in immunocompromised individuals. 